\title{
Herramienta para superar el dilema gerencial: Toma de decisiones o resolución de problemas
}

Recibido: 08/01/10 Aceptado: 21/01/10

El criterio vigente para enfrentar situaciones indeseadas es tomar decisiones en base a conceptos y paradigmas predefinidos, con lo que se "resuelve" un aspecto del problema, pero se genera fallas en otros aspectos, que devienen en problemas aún más graves que el original.

La resolución de problemas se maneja a nivel estratégico y se requiere un enfoque novedoso para enfrentar los problemas estratégicos.

Para esto, se emplea una matriz para la resolución de problemas complejos, el cual se construye mediante dos ejes cartesianos que definen cuatro dimensiones y cuatro campos de análisis. Esta herramienta permite analizar conjuntamente, la perspectiva del decisor en relación a su circunstancia de decisión.

Palabras clave: Dilema gerencial, toma de decisiones, resolución de problemas, matriz de resolución de problemas.

TOOL TO OVERCOME THE MANAGERIAL DILEMMA: DECISION MAKING OR PROBLEM SOLVING

\section{ABSTRACT}

The current approach to deal with unwanted situations is to make decisions based on concepts and pre-defined paradigms, this "solves" an aspect of the problem, but creates failures in other aspects which become even more serious problems than the original.

The resolution of problems is handled in a strategic level, it requires a change of approach to face reality.

For this, apply a tool that incorporates in a matrix array for solving complex problems, it is constructed by two Cartesian axes that define four dimensions and four analysis fields. This tool allows analyzed together, the perspective of the decision maker in relation to his environment decision.

KEY wORDS: Managerial dilemma, decision making, troubleshooting, troubleshooting matrix.
(1) Adolfo Acevedo Borrego

${ }^{(2)}$ Carolina Linares Barrantes

${ }^{(3)}$ Orestes Cachay Boza

\section{INTRODUCCIÓN}

Hasta tres cuartos del siglo veinte, los problemas de los sectores y las empresas se concentraban en la asignación de recursos, buscando la máxima eficiencia y productividad. A partir de los años setenta del siglo pasado, los criterios para el manejo de los sectores se han tornado inestables, la cambiante composición de los factores de producción, con la aparición de nuevos actores ha significado la aparición de inéditas constricciones que conlleva la necesidad de la introducción nuevos criterios para resolver los problemas de las empresas y los sectores.

\section{ENFOQUES PARA PERCIBIR LOS PROBLEMAS EMPRESA- RIALES}

En las empresas, la identificación de los problemas y su solución se basa en la aplicación de herramientas analítico-racionales que permiten analizar la situación, establecer las convenientes alternativas de mejora y tomar decisiones de elección de una u otra alternativa.

Para la percepción de los problemas empresariales, se consideran dos grandes enfoques.

\section{El enfoque racional}

Este enfoque estima que los problemas son únicos y su solución se deriva de la correcta aplicación de técnicas, principalmente cuantitativas. Es el enfoque de la dirección científica originada en el Taylorismo.

Se basa en los siguientes criterios:

$1^{\circ}$ Aplicación del método analítico (desagregar el problema en problemas de menor nivel).

1 Magíster en Administración, Ingeniero Industrial, Economista. Profesor en la Facultad de Ingeniería Industrial, Departamento de Producción y Gestión Industrial de la Universidad Nacional Mayor de San Marcos. E-mail: aacevedo@speedy.com.pe

2 Magíster en Dirección de Empresas por la Universidad de Piura, Ingeniero Industrial, estudios de Derecho. Profesora en la Facultad de Ingeniería de Sistemas e Informática de la Universidad Nacional Mayor de San Marcos. E-mail: klinares@speedy.com.pe

3 Magíster en Ingeniería Industrial, Ingeniero Industrial. Profesor en la Facultad de Ingeniería Industrial, Departamento de Producción y Gestión Industrial de la Universidad Nacional Mayor de San Marcos. E-mail: orestescachay@yahoo.es 
$2^{\circ}$ El problema es la definición del problema (problema definido, problema resuelto).

$3^{\circ}$ La solución se obtiene mediante métodos sistemáticos (algoritmos de solución).

$4^{\circ}$ No existe divergencia en la definición del problema ni en la solución (todos de acuerdo).

Los modelos de decisiones relacionados a este enfoque se encuentran en la ciencia de la administración (teoría de decisiones, IO), administración científica y gestión operaciones (productividad, resultados).

\section{El enfoque de sistemas}

Este enfoque considera que las empresas y su funcionamiento presentan mayor complejidad que la simple asignación de recursos. Se origina en los conceptos de la teoría general de sistemas de Von Bertalanfy, que estudia los modelos de comportamiento de los procesos biológicos y los amplía hacia los procesos empresariales.

Se basa en los siguientes criterios:

$1^{\circ}$ Las empresas son organizaciones sociales cuyos problemas de gestión se definen como difusos y "blandos" (problemas hard y problemas soft).

$2^{\circ}$ La búsqueda de soluciones debe considerar un rango de soluciones factibles, dentro de las cuales se ha de negociar consensualmente (opciones factibles y no factibles).

$3^{\circ}$ Se deben percibir los intereses en conflicto para encontrar las alternativas de mayor aceptabilidad (se identifican las opciones deseables y no deseables).

$4^{\circ}$ Diferenciar la resolución de problemas de la toma de decisiones.

Los modelos de decisiones relacionados al enfoque de sistemas se encuentran en la Teoría de sistemas, la metodología de los soft systems (sistemas blandos), la teoría de contingencias y el proceso estratégico (fuerzas, contingencias).

\section{DIFERENCIAS ENTRE TOMAR DECISIONES Y RESOLVER PROBLEMAS}

\section{Dilema entre tomar decisiones o resolver pro- blemas}

Dilema es la situación donde se ha de asignar recursos eligiendo entre varias alternativas. Al contrario del conflicto, que se refiere a la elección entre intereses contrapuestos con el objetivo de obtener la máxima satisfacción, el dilema se refiere a seleccionar entre cosas u objetos para obtener el máximo rendimiento.

Un tipo de dilema es diferenciar entre decidir y resolver. Tomar decisiones es diferente a resolver problemas, tiene un alcance mayor, plantea una visión divergente de la situación general. Resolver problemas es enfocado, tiene alcance concreto y plantea una visión convergente de un caso específico a solucionar.

Toda situación problemática implica una elección previa: decidir o resolver. Tomar decisiones implica elegir según paradigmas predefinidos, con el objetivo de mantener o cambiar, mejorar o empeorar la situación. Resolver problemas implica enfocarse en los resultados o efectos con el objetivo de cambiar y mejorar la situación. En otras palabras, el primero se enfoca en la elección aplicando paradigmas predefinidos, mientras el segundo se concentra en los efectos de las decisiones inadecuadas verificando las consecuencias en las dimensiones del mundo empresarial.

\section{Los marcos conceptuales para la toma de decisiones}

El axioma predominante para enfrentar situaciones indeseadas es tomar decisiones en base a conceptos y paradigmas que posee el decisor. La toma de decisiones se estudia bajo las cuatro grandes corrientes académicas, bajo el principio de la racionalidad analítica y la armonía de los objetivos de los actores.

En la escuela del proceso administrativo, se considera que las decisiones se enmarcan dentro de los conceptos de costos y ganancias, buscando lo más eficiente y el mayor rendimiento (Samuelson y Nordhaus, 2002). El criterio de decisión es el lucro.

En la escuela cuantitativa o estructuralista, se busca la aplicación de leyes de validez universal, en la empresa. Bajo la teoría de la decisión, se contempla la elección en condiciones de certeza, riesgo e incertidumbre (Krajewsky y Ritzman, 2000). El criterio de decisión es la optimización, maximización, minimización o mayor valor esperado.

En la escuela de las relaciones humanas y conductista se considera la racionalidad de las decisiones adoptadas bajo la teoría del rol de la personalidad y aprendizaje (Chiavenato, 2006). El criterio de decisión es la máxima satisfacción del hombre en la sociedad. 
Las diversas corrientes del cambio y la contingencia incluyen una diversidad de propuestas, como la teoría general de sistemas, teoría situacional, también la teoría de los juegos que introduce el efecto en los resultados (Chiavenato, 2006). El criterio de decisión es la solución de los conflictos si y solo si nos perjudican.

\section{PERSPECTIVA PARA LA RESOLUCIÓN DE PRO- BLEMAS EMPRESARIALES}

Bajo el enfoque de procesos, la empresa se considera un arreglo de actividades productivas, integradas por personas, ambiente, procesos y fuerzas, que se relacionan coordinadamente para recibir insumos del ambiente, transformarlos y producir bienes o servicios, valiosos y deseados por los clientes.

\section{Las dimensiones empresariales para la resolu- ción de problemas}

En toda actividad productiva intervienen dos dimensiones: la dimensión del ambiente y las presiones y la dimensión de la persona y sus actividades. En la actividad productiva empresarial participan cuatro elementos: individuos que ejecutan actividades sobre estructuras y materiales, de acuerdo a metas y presiones ambientales.

Estos elementos son los principios fundamentales que constituyen el cuerpo de la empresa, son las dimensiones persona, proceso, estructura y momento de la resolución de problemas.

1. El elemento humano. Es un factor activo o pasivo, es la persona con diversos grados de compromiso, respecto a los fines comunes de supervivencia, adaptación y crecimiento.

2. El elemento proceso. Comprende el trabajo y la organización productiva, es la autonomía para tomar decisiones, ejercer aptitudes y obtener logros que brindan satisfacción.

3. El elemento estructural. Se refiere al factor ambiental, los recursos, las instalaciones que conforman el marco físico y las fronteras de la tarea.

4. El elemento momento. Se refiere a las fuerzas de cambio o inacción que subyacen en toda labor en empresa.

La adecuada resolución de problemas ha de cubrir los cuatro elementos clave del mundo de la empresa: asignación eficiente de recursos, sistemas empresariales productivos, mantenimiento o mejora de los procesos de transformación y desarrollo y el efecto sobre las personas, como usuarios o como recursos. Se decide sobre el uso de los recursos empresariales, sean insumos, medios e instrumentos, se coordina el aprovisionamiento e ingreso al proceso productivo cuyo resultado son los bienes elaborados o los servicios prestados y cuyos beneficios han de dirigirse a las personas. Si los resultados no son los esperados, se toman decisiones de cambio y mejora, afectando al proceso, a la combinación de recursos y a la satisfacción de las personas.

\section{Perspectiva integral para la resolución de pro- blemas}

Las empresas son organizaciones sociales, cuyos problemas de funcionamiento operativo, orientación estratégica o dirección general se consideran como difusos y "blandos", por lo que la búsqueda de soluciones a los problemas debe considerar un rango de opciones correctas, dentro de las cuales se ha de negociar consensualmente para encontrar opciones deseables.

Se requiere un cambio de enfoque para enfrentar la realidad, o sea, la problemática del mundo empresarial. Para esto, se han de identificar las variables relevantes que interesan al decisor, las que se perciben holísticamente de manera que se pueden prever las fallas que surgen al implantar soluciones parciales o los nuevos problemas derivados de efectos imprevistos.

Los elementos para la toma de decisiones estratégicas son las dimensiones del mundo de la empresa y los campos de actuación en que se ubica el decisor, los que corresponden a la circunstancia empresarial y la perspectiva directiva (ver Gráfico 1).

Como se observa en el gráfico, los ejes x-y representan dos continuos cuyos extremos definen las cuatro dimensiones del mundo de las organizaciones: la dimensión estructural referida a las variables de los recursos físicos y la inversión, la dimensión del proceso referida a las variables de transformación industrial, la dimensión del momento y cambio referida a la tecnología y desarrollo, la dimensión humana referida a las personas y los grupos sociales afectados o beneficiados por la acción empresarial.

La intersección de los ejes definen cuatro campos de actuación. El campo A es el área del logro y la actividad económica, es el empleo productivo de los recursos y la toma de decisiones sobre recursos y personas. El campo B es el área del poder y fijación de roles y posiciones dentro de la estructura so- 
Gráfico 1. Elementos para la toma de decisiones estratégicas: dimensiones y campos.

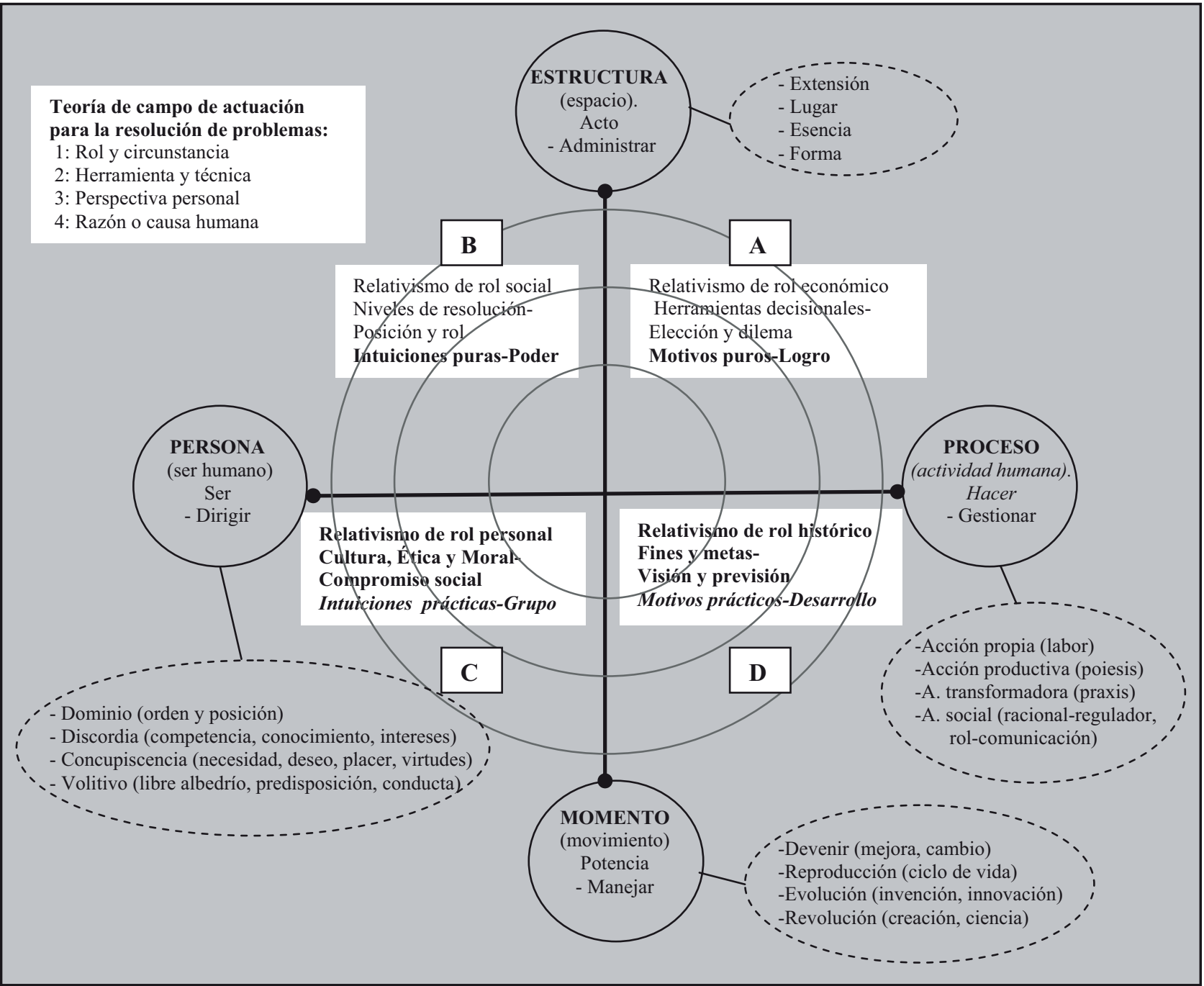

Fuente: Acevedo, Adolfo. "Modelo 4D del mundo de la empresa". Paper, UNMSM-FII. Perú.

cioeconómica, es el efecto social de las decisiones económicas. El campo $\mathrm{C}$ es el área de la afiliación y el compromiso personal, es la conducta dentro de las fronteras establecidas por la cultura, las reglas. El campo D, el desarrollo y la visión del futuro, es la preparación del orden o el cambio, de la estabilidad o la mejora.

\section{Pautas a considerar en la resolución de problemas}

Para resolver situaciones de empresa, se ha de tener en cuenta los siguientes puntos:

$1^{\circ}$ Definir el límite del problema, o sea, qué se quiere resolver (el sector, la empresa, el negocio, una función, una tarea, problemas de personas).

$2^{\circ}$ Definir el nivel de resolución del problemas (estratégico, táctico, operativo). $3^{\circ}$ Prever los efectos probables de la ejecución de la elección tomada (efectos colaterales en personas, en las tareas, en la organización o en la tecnología).

$4^{\circ}$ La manera de identificar las variables de las metas (mercado, producto, funciones, tiempo).

$5^{\circ}$ Capacidad para percibir la manera en que las partes afectadas asumirán la implantación de las decisiones.

$6^{\circ}$ Considerar que las decisiones las toman las personas y que existen diferentes factores que pueden sesgar las opciones a considerar.

Básicamente, la solución de la problemática de empresa implica seguir la secuencia de indagación y solución de problemas a partir de la observación empírica del mundo real, identificando las deficiencias y superándolas para llevarlo a lo deseado. Ya 
que, al final, no se trata de encontrar soluciones óptimas, sino alcanzar soluciones factibles técnicamente, deseables por los grupos involucrados y sostenibles en el tiempo.

\section{HERRAMIENTA PARA LA RESOLUCIÓN DE PROBLEMAS COMPLEJOS}

Cuando se intenta dar solución a una situación empresarial, el caso se puede plantear desde diferentes posiciones, a partir de definición que en toda actividad productiva intervienen cuatro elementos: las personas, el medio ambiente de trabajo, las actividades a ejecutar y las presiones: internas para alcanzar metas y externas de la competencia.

\section{Un renovado enfoque estratégico para afrontar la resolución de problemas}

El proceso de resolver problemas parte de dos opciones liminares: la primera se refiere a elegir o no elegir, la segunda se refiere a los criterios de decisión: positivismo racionalista, pragmatismo e intuición, contingencias y político.

1) Criterio analítico-racional. Se orienta hacia la solución óptima, cuya máxima se resume en "Problema definido, problema resuelto". Utiliza el método científico para el análisis y la solución de problemas. Este criterio es adecuado para el diseño de sistemas y el control.

2) Criterio pragmático-intuitivo. Considera el bagaje de experiencia personal y elementos poco definibles y de "pálpito". Utiliza el sentido común y la intuición. Este criterio es recomendable ante situaciones estratégicas y problemas blandos y difusos.

3) Criterio de contingencia y político. Considera los intereses y presión de diversos grupos confron- tados. Este criterio es frecuente en aquellas organizaciones cuyo desempeño y resultados son irrelevantes.

Se requiere un viraje en el enfoque para enfrentar la problemática del mundo empresarial, para esto, se aplica una herramienta que integra, en un arreglo matricial, las variables relevantes de la situación, de manera que se perciben integralmente y es posible prever los problemas adicionales que surgirían ante soluciones parciales.

\section{Matriz de resolución de problemas complejos}

La matriz de resolución de problemas complejos es una herramienta estratégica para analizar situaciones problemáticas, prevenir efectos indeseados y plantear opciones equilibradas, en función a los intereses de los actores. En el gráfico 2, se muestra la matriz de resolución de problemas complejos, que considera la relación entre las diferentes dimensiones y analiza sus efectos mediante los cuatro campos.

La resolución de problemas se maneja a nivel estratégico, ya que la alternativa que se elija ha de tener efectos en los diversos campos de actuación el decisor, se puede emplear una simbología como la del cuadro 1.

Los posibles efectos (positivos o negativos) habrán de orientar las líneas de acción y las políticas empresariales, de manera que se establezca el adecuado marco para las áreas de decisión y para la actividad operacional.

\section{Análisis de los campos}

Los criterios para analizar los cuatro campos son los siguientes:

Cuadro 1. Efecto de la decisión en las variables empresariales

\begin{tabular}{|c|l|l|}
\hline Símbolo & \multicolumn{1}{|c|}{ Descripción } & \multicolumn{1}{c|}{ Comentario } \\
\hline $\mathrm{dk}$ & Efecto positivo & Implantar \\
\hline $\mathrm{X}$ & Efecto negativo & Redefinir \\
\hline$¿ ?$ & Se desconoce sus consecuencias & Analizar si fuere pertinente \\
\hline$i !$ & Resultados imprevisibles & Requiere mayor estudio \\
\hline$@$ & Se potencia con la tecnología & Verificar externalidades \\
\hline- & Indiferente. No interactúan & Verificar consistencias \\
\hline
\end{tabular}

Fuente: Elaboración propia. 
Gráfico 2. Matriz de resolución de problemas estratégicos

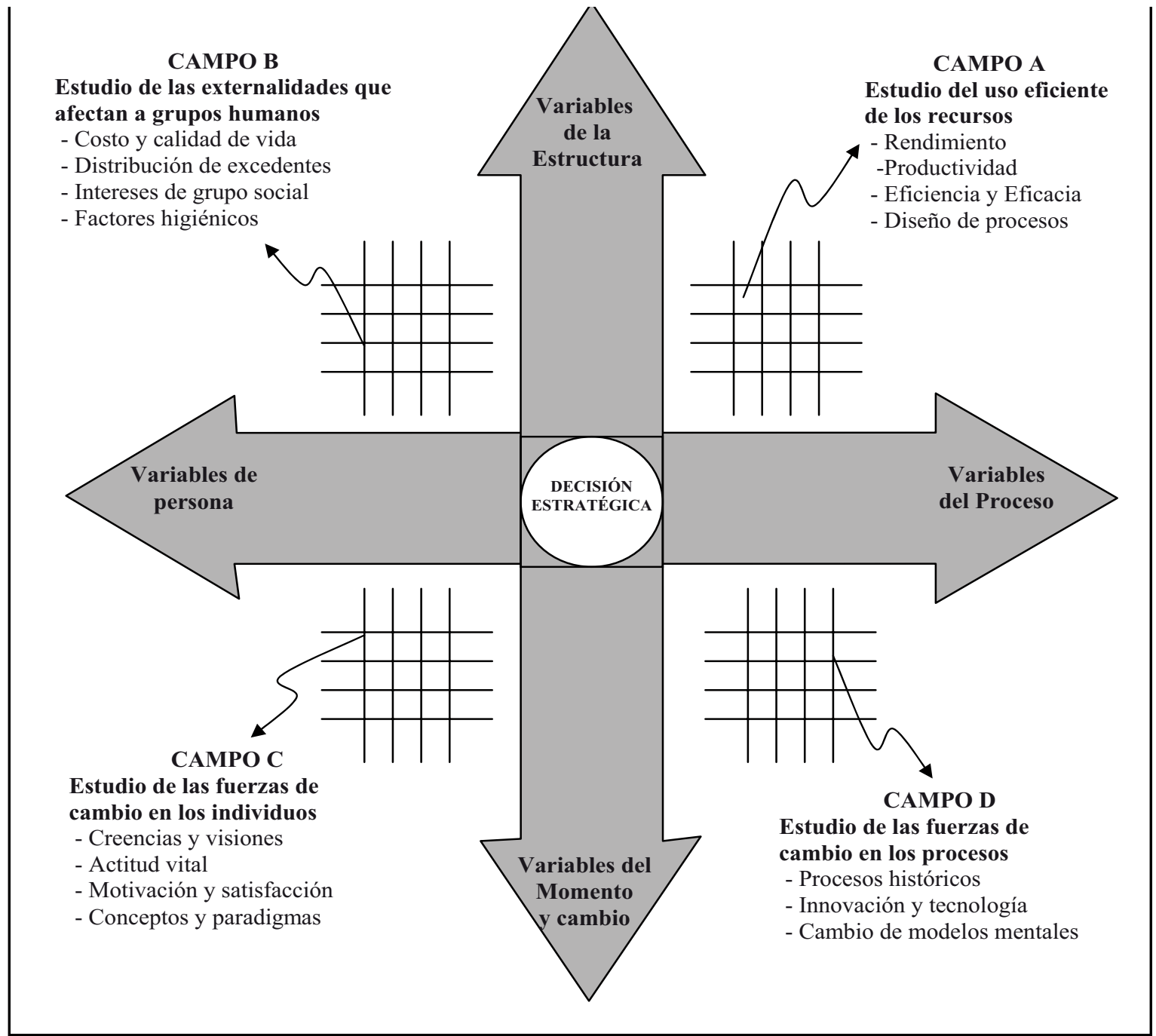

Fuente: Acevedo, Adolfo. "Modelo 4D del mundo de la empresa". Paper, UNMSM-FII. Perú.

1) Campo A. Efecto del ambiente físico, instalaciones y recursos sobre la eficiencia y productividad del proceso de producción. Las empresas enfocadas en este campo decisional son aquellas cuyo core productivo son las actividades primario-extractivas. Básicamente, sus decisiones deben considerar una amplia gama de efectos, desde la eficiencia y productividad de la operación en sí y los efectos colaterales derivados de la modificación de medio ambiente y la extracción de recursos.

2) Campo B. Del efecto del entorno y uso de recursos en el ambiente social y calidad de vida. Las empresas de alta interacción con el medio ambiente cuyos efectos son relevantes en los grupos sociales, deben priorizar sus relaciones con las co- munidades que se perciben como afectadas por la manipulación del ambiente físico y los recursos.

3) Campo C. De las fuerzas sociales e intereses y su influencia sobre la sociedad. Considera las decisiones sobre innovación pero que se potencia o se retrasa por el perfil del personal. La menor especialización del personal puede limitar la introducción de tecnologías de avanzada, ya que el cambio e innovación se apoya en el conocimiento.

4) Campo D. De la innovación tecnológica y su influencia sobre los procesos productivos. Considera las decisiones que afectan a los procesos, productos o servicios de alta o baja intensidad en tecnología. Este campo es muy importante para las empresas enfocadas en las innovaciones tecnológicas. 


\section{CASO EJEMPLO: CAMBIO EN EL MODELO EM- PRESARIAL ESTATAL}

En la década de los noventa, se empezó a cuestionar a la actividad empresarial del Estado. En el Perú, el aparato productivo de propiedad del Estado representó parte sustantiva en la actividad económica, captando inversión, interrelacionando sectores y dinamizando el mercado; pero sus problemas de contracción e incapacidad para generar excedentes se constituyeron en un cuello de botella que dificultó el desenvolvimiento empresarial y el desarrollo de otros sectores industriales. A partir del fracaso de la participación estatal en la economía, se promueve la privatización de las empresas públicas como la solución definitiva para el subdesarrollo y para modernizar las economías atrasadas.

La privatización fue definida como un proceso de transferencia de propiedad y gestión de las empresas estatales, formando parte de los programas de estabilización, liberalización y desregulación de los sectores económicos de los países con deficiencias estructurales, ineficiencia y baja productividad en sus empresas, como era y es el caso de América Latina.

A la privatización, se le considera un tema del nivel estratégico orientado a la supervivencia y al crecimiento de las organizaciones públicas con problemas financieros y con un modelo de desarrollo agotado y en retroceso.

\section{LA SITUACIÓN PROBLEMA INICIAL}

Dentro de los grupos de interés, algunos enuncian que la privatización es una condición imperativa para evitar el crecimiento del aparato estatal, para aumentar las inversiones productivas y para reducir la deuda externa, eliminando definitivamente las distorsiones de mercado; otros consideran que este proceso representa un retroceso de dos décadas en el avance económico de los países, ya que ha originado mayor dependencia de capitales externos -públicos y privados-, ha reducido la capacidad de manejo de los recursos de los actores nacionales, ha debilitado el poder político local, ha distorsionado y casi eliminado los mecanismos de libre mercado.

\section{La matriz de resolución de problemas estratégicos}

Esta matriz es un modelo gráfico que considera la relación entre las diferentes dimensiones y analiza los efectos integrales de la privatización en los cuatro campos. En el Gráfico 3, se muestran las variables relevantes (preliminares y a modo de ejemplo) para el análisis de la decisión de privatización.

\section{Análisis general de la propuesta}

El análisis de la propuesta consideró los efectos en las variables, dentro de los cuatro campos de actuación. El Campo A relaciona el ambiente físico y su efecto sobre el proceso de producción, principalmente si la decisión eleva o reduce las restricciones y cuellos de botellas no empresariales que impidan o retrasen el funcionamiento de los procesos productivos. En el Campo B, la preocupación de los decisores se enfoca en las relaciones y en la información ofrecida a los grupos afectados o beneficiados por la actividad productiva privatizada. En el Campo C, se prevé que las decisiones de cambio tienden a priorizar la inversión en tecnologías de automatización de los procesos para reducir la dependencia de las personas, estabilizar el flujo de los procesos, reducir costos, y obtener mayor control sobre los procesos y menos sobre las personas. El Campo D considerará que el perfil del personal de las nuevas empresas ha de ser de especialista focalizado en tareas muy concretas y de especialidades diversas. En varios países de Sudamérica, el sector estatal de telecomunicaciones ha mostrado mayor crecimiento que sus equivalentes privatizados de otros países, lo que ha cuestionado la validez de la privatización como única opción de mejora de las economías nacionales.

\section{Los resultados derivados del empleo de la matriz}

Las empresas estatales estuvieron inmersas en un proceso de cambio turbulento que modificó su propiedad y comportamiento.

Los sectores, antes dominados por las grandes empresas públicas, se dirigieron hacia el manejo privado, la mayor competencia y la gestión competitiva enfocada en resultados financieros.

La tendencia derivó en la consolidación de empresas mundiales y globalizadas, con amplia libertad de decisión y acción y con un cambio radical en la gestión, frente a entornos impredecibles y poco amigables. Los decisores asumen decisión sobre la adopción de tecnologías, a la vez que controlan el efecto de los avances tecnológicos sobre el grado de automatización de los procesos productivos.

\section{CONCLUSIONES}

Existen diferencias conceptuales entre tomar decisiones y resolver problemas. El criterio vigente para enfrentar situaciones indeseadas es tomar decisiones en base a conceptos y paradigmas prede- 
Gráfico 3. Matriz de resolución de problemas estratégicos

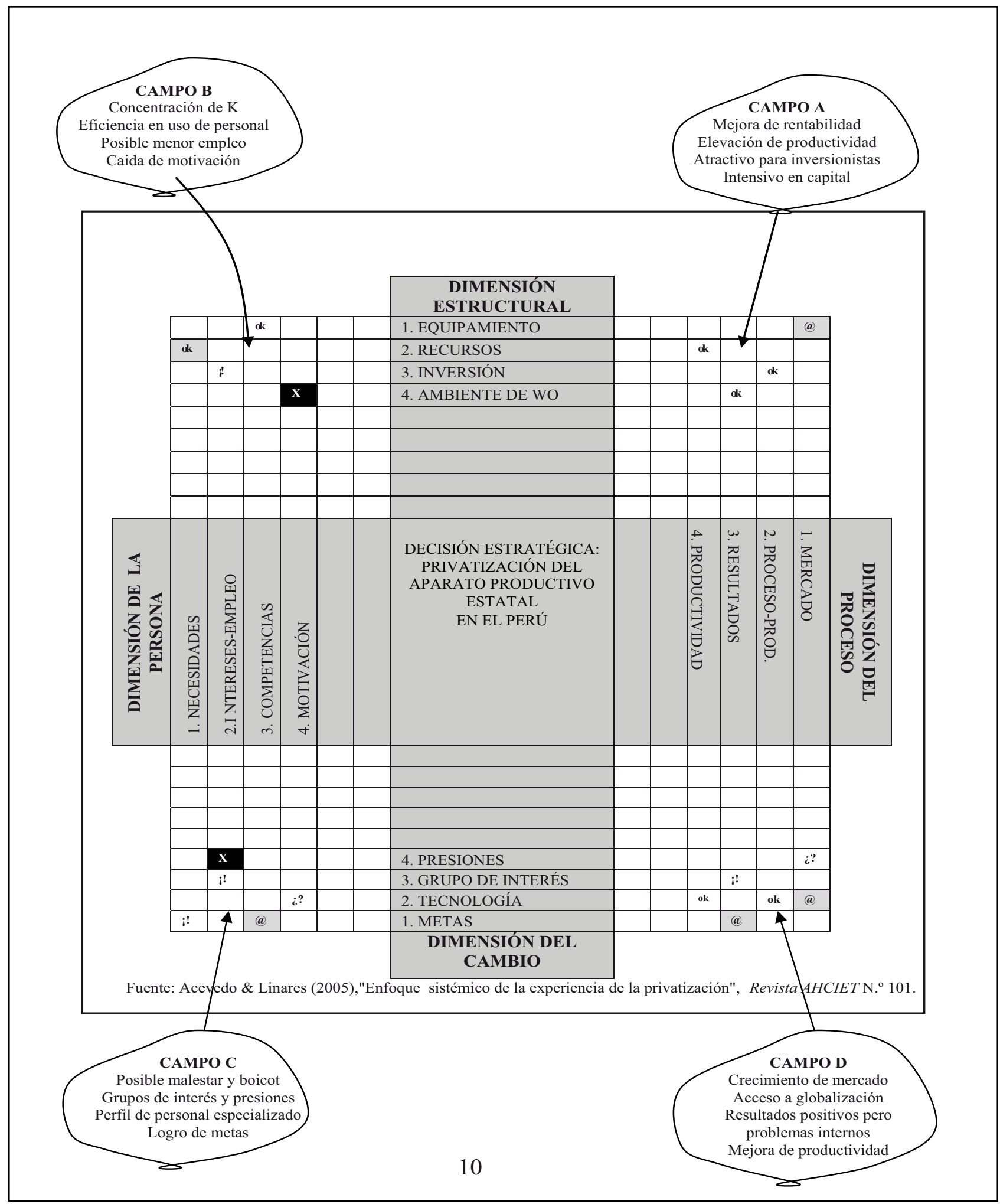

Fuente: Adaptado de Acevedo \& Linares (2005),"Enfoque sistémico de la experiencia de la privatización", Revista AHCIET. 
finidos, lo que "resuelve" un aspecto del problema, pero genera fallas en otros aspectos que devienen en problemas aún más graves que el original.

Se requiere un cambio de enfoque para enfrentar la realidad. Para esto, se aplica una herramienta que integra, en un arreglo matricial, las variables relevantes de la situación, de manera que se puede prever los problemas adicionales que surgirían ante soluciones parciales. Así, la resolución de problemas se maneja a nivel estratégico.

La matriz para la resolución de problemas complejos se construye mediante dos ejes cartesianos que contienen las cuatro dimensiones del mundo de la empresa: la dimensión estructural de las instalaciones, inversiones y organización, la dimensión del momento, el cambio y la innovación tecnológica, la dimensión del proceso y la transformación productiva de los recursos en productos, y la dimensión de la persona referido a las necesidades, competencias y motivaciones humanas. Estas dimensiones la definen cuatro campos de análisis: el campo A referido al efecto del ambiente sobre la eficiencia y productividad, el campo B sobre el efecto del uso de los recursos en la sociedad, el campo $\mathrm{C}$ sobre las fuerzas de cambio tecnológico y su influencia en la calidad de vida laboral, y el campo D referido a la innovación y su influencia en los procesos productivos.

La matriz de resolución de problemas complejos es una herramienta de análisis estratégico que considera la relación entre las diferentes dimensiones de la empresa y analiza los efectos integrales en los cuatro campos, de manera que se puede prever las consecuencias deseadas y las externalidades que reducen la eficacia de las decisiones. Esta herramienta matricial sintetiza visiones integradoras y facilita que la resolución de problemas se maneje a nivel directivo, mediante la definición de variables conceptuales e ideológicas.

El valor de herramienta se ubica en la posibilidad de entender los criterios sesgados de decisión y los marcos conceptuales parciales del decisor, de esta manera se evitan las soluciones "óptimas" de las herramientas cuantitativas, los enfoques analítico-racionales que consideran que existe una mejor solución, y el enfoque de tomar decisiones en base a conceptos y paradigmas predefinidos.

En suma, se intenta resolver el aspecto clave del problema en cuestión, previendo la generación de fallas en otros aspectos y evitando nuevos problemas, que complican aún más, la situación problemática.

\section{REFERENCIAS BIBLIOGRÁFICAS}

1. Acevedo Borrego, Adolfo (2003). El modelo de los sistemas sociotécnicos en la teoría y praxis empresarial. Proceedings XXXVIII Asamblea Anual CLADEA. Perú.

2. Acevedo Borrego, Adolfo (1992). Planeamiento estratégico en telecomunicaciones. Universidad del Pacífico. Perú.

3. Acevedo, Adolfo Oswaldo (2009). Modelo 4D del mundo de la empresa. Paper, UNMSM- FII. Perú.

4. Acevedo Borrego, Adolfo \& Linares Barrantes, Carolina (2005). "Enfoque sistémico de la experiencia de la privatización", Revista AHCIET No.101.

5. Blair \& Whitson (1973). Elementos de ingeniería de sistemas industriales. Editorial Prentice-Hall Internacional. España.

6. Chiavenato, Idalberto (2004). Introducción a la teoría general de la administración. $7^{\mathrm{a}}$ ed. Edit. Mc Graw Hill. México.

7. Hammond, Keeney y Raiffa (1999). Decisiones inteligentes. Editorial Norma. Colombia.

8. Hammond, Keeney y Raiffa (2002). Las trampas ocultas en la adopción de decisiones. Editorial Deusto. Colombia.

9. Handy, Charles (1997). "Encontrar sentido en la incertidumbre" en Repensando el futuro. Editado Rowan Gibson. Colombia.

10. Harvard Business Review (2002). La toma de decisiones. Editorial Deusto. Colombia.

11. Hill, Gareth, Jones (1996). Administración estratégica un enfoque integrado, Edit. Mc Graw-Hill/ Interamericana. México.

12.Hodson, William (1996). Maynard. Manual del ingeniero industrial. 4. ${ }^{\mathrm{a}}$ ed. Edit. Mc Graw-Hill, México.

13.Katz \& Rosenzweig (1988). Administración en las organizaciones, enfoque de sistemas y de contingencias. Ed. Mc Graw-Hill, México.

14.Krajewsky y Ritzman (2000). Administración de operaciones. $5 .^{a}$ ed. Pearson-Prentice Hall. México.

15. Kuhn, Thomas (1971). La estructura de las revoluciones científicas. Fondo de Cultura Económica. México. 
16. León, Orfelio (2001). Tomar decisiones difíciles. 2. ${ }^{a}$ ed. Editorial Mc Graw-Hill. España.

17. Linares, Carolina (2005). Nota Técnica sobre innovación tecnológica en empresas de servicios. Proceedings Cladea 2005. Universidad de Chile.

18. Minztberg, Quinn y Voyer (1997). El proceso estratégico conceptos, contextos y casos. Edit. Prentice Hall. México.

19. Samuelson y Nordhaus (2002). Economía. 17. ${ }^{a}$ ed. Edit. Mc Graw-Hill. España.
20. Thompson y Strickland (1998). Dirección y administración estratégicas. Edit. Mc Graw-Hill. México.

21. Vaill, Peter (1967). "La ingeniería industrial y los sistemas sociotécnicos”. En GROFF Y MUTH. Sistemas de producción. Edit. El Ateneo. Buenos Aires.

22. Zandin, Kjell (2005). Maynard. Manual del ingeniero industrial. 2. ${ }^{a}$ ed. Edit. McGraw-Hill, México. 